\title{
Binding Energy Of A Donor In A Spherical Quantum Dot , GaAs
}

\author{
S.R.Chitra., M.Sc., M.Phil., PGDCA.,[Ph.D]., \\ [working as Lecturer in Physics, @ N.P.R College in Natham, Dindigul Dt and doing Ph.D., at Anna \\ University, Thiruchirappalli, Tamil Nadu in India]
}

\begin{abstract}
The binding energy of a donor in spherical quantum dots $(Q D s)$ is calculated, using a variational approach within the effective mass approximation. The binding energy is computed for GaAs $Q D$ as a function of the dot size for different impurity positions, and also as a function of the impurity position for different dot sizes. The results of mine show that when the impurity binding energy increases with the reduction in the dot dimension. The binding energy is also found to depend on the location of the impurity, and the same is the maximum for the on-center impurity. Also I found that the value of the polarizability obtained is several orders higher than the hydrogen atom value.
\end{abstract}

Keywords: Spherical quantum dot, Donor binding energy, Impurity state, Parabolic confinementQuantum dots, quantum wells gallium arsenide, III-V semiconductors, impurity states, effective mass, donor Binding, Semiconductor compounds

PACS 73.20.Dx, 73.20.Hb, 73.21. La $, \underline{73.21 .-b}, \underline{73.61 . E y}, \underline{73.20 . \mathrm{Hb}}, \underline{71.18 .+\mathrm{y}}, 71.38 .+\mathrm{I}, 71.20 . \mathrm{Nr}$

\section{I . Introduction.}

The work on the binding energy of a donor atom within an infinite potential well initiated several studies on the impurity states in quantum wells. Similar studies have also been extended for structures with lower dimensionality, such as quantum-well wires (QWWs) and quantum dots (QDs). The study of low dimensional systems has received much attention in recent years, especially due to the discovery of such effects as the quantum Hall Effect in two - dimensional (2D) systems. The physics or even lower dimensional systems present intriguing challenges both theoretically and experimentally. With the development of modern technology, it is now possible to produce (Quasi-) 0D systems that confine electrons in all three spatial dimensions. In this communication, we shall study the effect of polarizability and the binding energy of donor in a spherical QD of a wide gap material; the derived results will be computed for GaAs QD. The binding energy will be computed as a function of the dot dimension for different impurity positions, and also as a function of the impurity position for different dot sizes.

\section{II . Theory.}

In the effective mass approximation, the Hamiltonian of a single hydrogenic impurity in a spherical QD with parabolic confinement can be written as

$\mathrm{H}=\left[\mathrm{P}^{2} / 2 \mathrm{~m} *\right]+1 / 2\left[\mathrm{~m} * \omega^{2} \mathrm{r}^{2}\right]-\mathrm{e}^{2} / \varepsilon\left|\left(\mathrm{r}-\mathrm{r}_{\mathrm{i}}\right)\right|$

Where e and $\mathrm{m}^{*}$ are, respectively, the electronic charge and effective mass, $\mathrm{P}$ is a momentum, $\omega$ is a characteristic frequency, $\varepsilon$ is the dielectric constant of the dot material, and $\mathrm{r}_{\mathrm{i}}$ gives the location of the impurity with respect to the center of the dot.

In order to calculate the ground state of the impurity binding energy, the variational technique is used, and for this the trial wave function is taken as

$\psi(\mathrm{r})=\mathrm{N}(\lambda) \exp \left(-\beta \mathrm{r}^{2} / 2\right) \exp \left(-\lambda\left|\left(\mathrm{r}-\mathrm{r}_{\mathrm{i}}\right)\right|\right)$,

Where $\beta=m^{*} \omega / \mathrm{h} / 2 \pi$. constant.

Here $h$ being the Planck's constant. $\lambda$ is the variational parameter and $N(\lambda)$ is the normalization

The ground state energy of the hydrogenic impurity is worked out from the above equations by using the below relation.

$\mathrm{E}\left(\lambda, \beta, \mathrm{r}_{\mathrm{i}}\right)=\left\langle\psi^{*} / \mathrm{H} / \psi\right\rangle /\left\langle\psi^{*} / \psi\right\rangle$

Here $\psi^{*}$ means the conjugate of the eigen function $\psi$.

Our work consists of two cases.

That is, we derived the binding energy for two cases:

I. $\quad r_{i}=0$., ie., the location of the impurity is zero.

II. $r_{i}=a$.r., ie., impurity at the inner surface of the dot.

Cases: 


\section{Case 1:}

At $r_{i}=0$, the location of the impurity is zero.

Therefore the Hamiltonian of a single hydrogenic impurity in a spherical QD with parabolic confinement can be written as

$\mathrm{H}=\left[\mathrm{P}^{2} / 2 \mathrm{~m}\right]+1 / 2\left[\mathrm{~m}^{*} \omega^{2} \mathrm{r}^{2}\right]-\mathrm{e}^{2} / \varepsilon\left|\left(\mathrm{r}-\mathrm{r}_{\mathrm{i}}\right)\right|$

The trial wave function is taken as

$\psi(\mathrm{r})=\mathrm{N}(\lambda) \exp \left(-\beta \mathrm{r}^{2} / 2\right) \exp \left(-\lambda\left|\left(\mathrm{r}-\mathrm{r}_{\mathrm{i}}\right)\right|\right)$,

Where $\beta=\mathrm{m}^{*} \omega / \mathrm{h} / 2 \pi$.

Here $h$ being the Planck's constant. $\lambda$ is the variational parameter and $N(\lambda)$ is the normalization constant.

Normalization condition:

$\langle\psi * / \psi\rangle=1$

By equating everything, we get

$\int \psi(\mathrm{r})\left[1 / 2 \mathrm{~m}^{*} \omega^{2} \mathrm{r}^{2}\right] \psi(\mathrm{r}) \mathrm{d} \tau=2 \pi \mathrm{N}^{2} \mathrm{e}^{2} \mathrm{e}^{\lambda / \beta} / \mathrm{m}^{*}\left[\lambda(\operatorname{sqrt}(\pi)) / \beta^{3 / 2-} 1 / 2 \beta\right]$

This is the final solution for case (i).

Then the binding energy will be,

$<\mathrm{E}>=2 \pi \mathrm{N}^{2} \mathrm{~h}^{2} \mathrm{e}^{\lambda / \beta} / \mathrm{m}^{*}\left\{-5(\operatorname{sqrt}(\pi)) / 2 \beta^{1 / 2}\right\}-$

By using this the binding energy was computed for GaAs QD as a function of the dot size. The results are tabulated in Table:

Table:

\begin{tabular}{|l|l|l|l|}
\hline $\boldsymbol{\beta}^{-\mathbf{1 / 2}} \quad(\mathbf{n m})$ & $\lambda \quad \begin{array}{l}\langle\mathbf{E}>\text { in Ryd* } \\
(\mathbf{P r e s e n t})\end{array}$ & $\begin{array}{l}\langle\mathbf{E}>\text { in Ryd* } \\
(\text { Reference) }\end{array}$ \\
\hline 2 & $0.47 * 10^{5}$ & 13.18 & 13.1 \\
\hline 4 & $0.46^{*} 10^{5}$ & 12.10 & 12.0 \\
\hline 6 & $0.44 * 10^{5}$ & 7.93 & 7.4 \\
\hline 8 & $0.42 * 10^{5}$ & 4.99 & 4.5 \\
\hline 10 & $0.41 * 10^{5}$ & 3.18 & 3.0 \\
\hline
\end{tabular}

*1 Ryd $=5.3 \mathrm{meV}$ for GaAs

In this the present works are compared with the values of the references . Finally a graph is drawn between the impurity binding energy versus the dot size.

\section{Graph:}

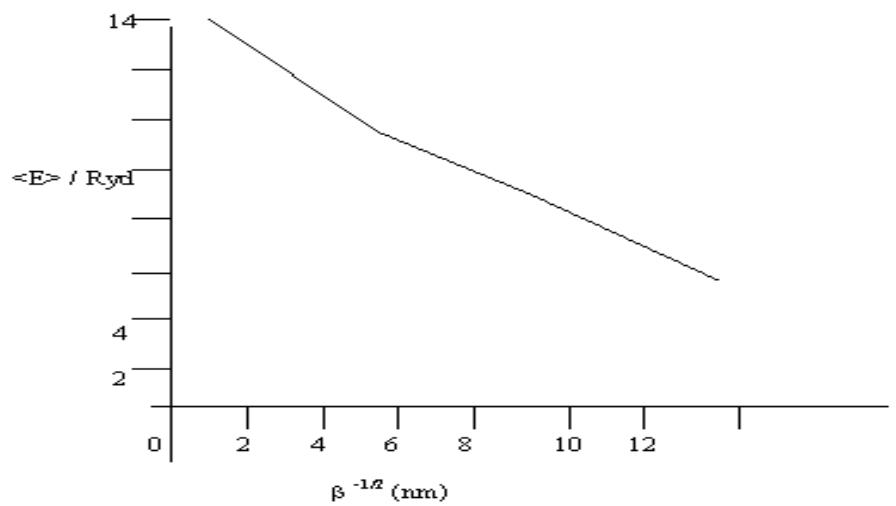

For the case $r_{i}=0$. From this figure it follows that the donor binding energy decreases as the dot size increases. As the size $-\rightarrow \infty$, the energy should 1 Ryd.

The impurity binding energy in spherical GaAs QDs with parabolic confinement versus the dot size.

\section{Case 2:}

At $r_{i}=a$, the location of the impurity is at the inner surface of the dot.

Therefore the Hamiltonian of a single hydrogenic impurity in a spherical QD with parabolic confinement can be written as 
$\mathrm{H}=\left[\mathrm{P}^{2} / 2 \mathrm{~m} *\right]+1 / 2\left[\mathrm{~m} * \omega^{2} \mathrm{r}^{2}\right]-\mathrm{e}^{2} / \varepsilon|(\mathrm{r}-\mathrm{a})|$

The trial wave function is taken as

$\psi(\mathrm{r})=\mathrm{N}(\lambda) \exp \left(-\beta \mathrm{r}^{2} / 2\right) \exp (-\lambda|(\mathrm{r}-\mathrm{a})|)$,

Where $\beta=m^{*} \omega / \mathrm{h} / 2 \pi$.

the normalization constant.

Here $h$ being the Planck's constant. $\lambda$ is the variational parameter and $N(\lambda)$ is

Normalization condition: $\langle\psi * / \psi\rangle=1$

By equating everything, we get

$\int \psi(\mathrm{r})\left[1 / 2 \mathrm{~m}^{*} \omega^{2} \mathrm{r}^{2}\right] \psi(\mathrm{r}) \mathrm{d} \tau=2 \pi \mathrm{N}^{2} \mathrm{e}^{2} \mathrm{e}^{\lambda / \beta} / \mathrm{m}^{*}\left[\lambda(\operatorname{sqrt}(\pi)) / \beta^{3 / 2-}(2 \lambda / \beta)+(1 / 2 \beta)-\ldots ..\right]$

This is the final solution for case (i).

Then the binding energy will be,

$\langle\mathrm{E}\rangle=2 \pi \mathrm{N}^{2} \mathrm{~h}^{2} \mathrm{e}^{\lambda / \beta} / \mathrm{m}^{*}\left\{25(\operatorname{sqrt}(\pi)) / 8 \beta^{1 / 2}\right\}-$

By using this the binding energy was computed for GaAs QD as a function of the dot size. The results are tabulated in Table:

Table:

\begin{tabular}{|l|l|l|l|}
\hline $\boldsymbol{\beta}^{-\mathbf{1} / \mathbf{2}}(\mathbf{n m})$ & $\lambda \quad \begin{array}{l}\langle\mathbf{E}>\text { in Ryd* } \\
(\text { Present })\end{array}$ & $\begin{array}{l}\langle\mathbf{E}>\text { in Ryd* } \\
\text { (Reference) }\end{array}$ \\
\hline 2 & $0.46^{*} 10^{5}$ & 12.28 & 12.0 \\
\hline 4 & $0.45 * 10^{5}$ & 11.48 & 10.9 \\
\hline 6 & $0.42 * 10^{5}$ & 7.10 & 6.9 \\
\hline 8 & $0.40 * 10^{5}$ & 4.21 & 4.0 \\
\hline 10 & $0.39 * 10^{5}$ & 3.16 & 3.08 \\
\hline
\end{tabular}

*1 Ryd = $5.3 \mathrm{meV}$ for $\mathrm{Ga}$ As

In this the present works are compared with the values of the references .

Finally a graph is drawn between the impurity binding energy versus the dot size. For the case $r_{i}=$ a. From this figure it follows that the donor binding energy decreases as the dot size increases. As the size $\rightarrow \infty$, the energy should 1 Ryd.

The impurity binding energy in spherical GaAs QDs with parabolic confinement versus the dot size.

From Table II, it follows that as in the previous case, the binding energy decreases when the dot size increases.

Comparisons of Table I \& II reveals that the binding energy is larger in the case where the impurity is at the centre of the dot. The graph is drawn between the beta values and the energy values.

\section{Graph:}

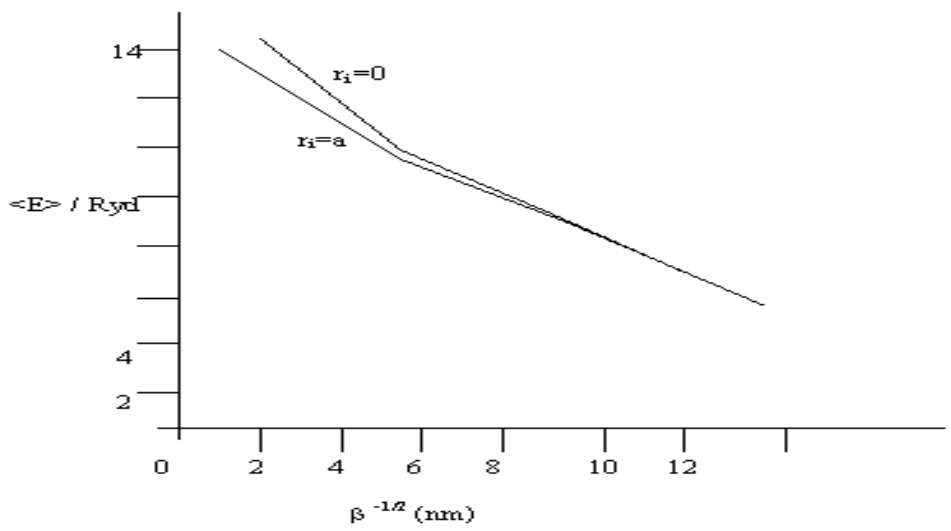

size.

The impurity binding energy in spherical GaAs QDs with parabolic confinement versus the dot 
\#include $<$ stdio.h $>$

\section{Program for calculating Binding Energy.}

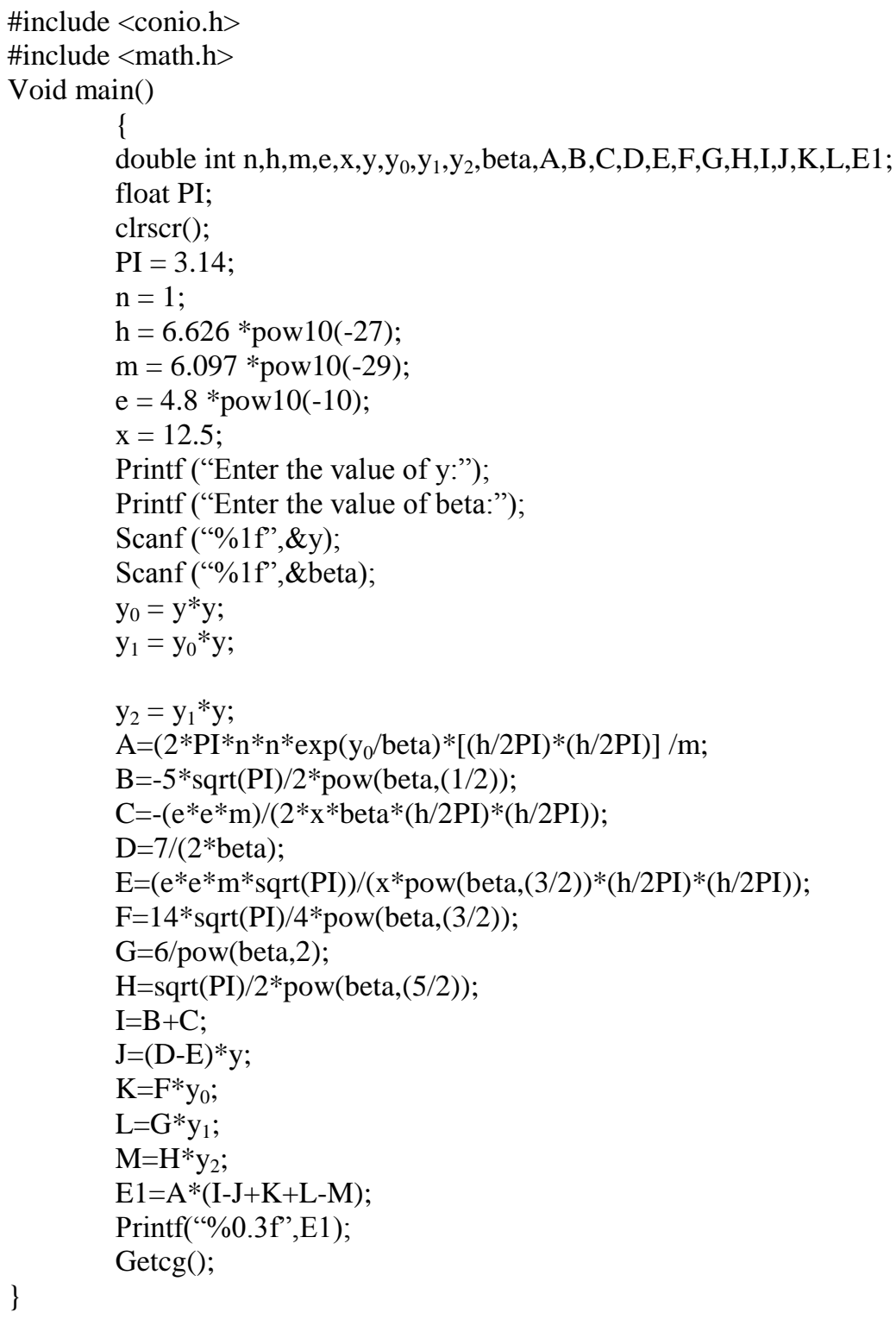

IV. Polarizability with an effect of an Electric field.

The donor atom in our system in an external electric field is given by, $\mathrm{H}=\left[\mathrm{P}^{2} / 2 \mathrm{~m}\right]+1 / 2\left[\mathrm{~m}^{*} \omega^{2} \mathrm{r}^{2}\right]-\mathrm{e}^{2} / \varepsilon\left|\left(\mathrm{r}-\mathrm{r}_{\mathrm{i}}\right)\right|+\varepsilon \mathrm{ez}$

We use the trial wave function, $\psi(\mathrm{r})=\mathrm{N}(\lambda) \exp \left(-\beta \mathrm{r}^{2} / 2\right) \exp (-\lambda|(\mathrm{r}-\mathrm{a})|)(1+\alpha \mathrm{ez})$ where $\mathrm{z}=\operatorname{rcos} \vartheta$. And $\alpha$ is treated as a variational parameter.

In the case of 'On- Centre ' impurity $\left(\mathrm{r}_{\mathrm{i}}=0\right)$, the normalization condition yields, $\mathrm{N}^{2}=1 / 4 \pi \mathrm{e}^{\lambda / \beta}\left[\left[\operatorname{sqrt}(\pi) / \beta^{3 / 2}(\ldots)-\lambda / \beta\left[1+\left(\varepsilon^{2} \alpha^{2}\right) / 3\right]+\left(\lambda^{2} / \beta^{5 / 2}\right)(\ldots)-\ldots ..\right]\right.$

If we put $\alpha=0$, then we get the $\mathrm{N}^{2}$ value for case 1 . (i.e., $\mathrm{r}_{\mathrm{i}}=0$ )

I simplified this for second case also and I found out the expectation value of the Hamiltonion and also the binding energies were computed for different values of the electric fields. The results are presented in the Table. 


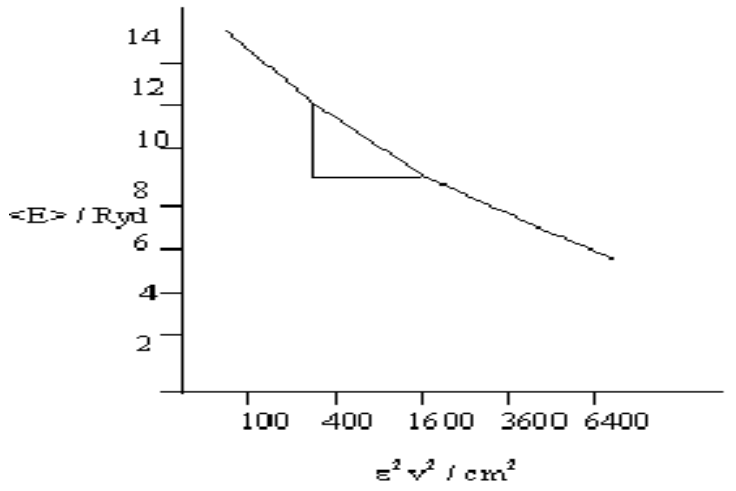

Using these datas, a graph was drawn for binding energy $\mathrm{Vs} \varepsilon^{2}$, (see in figure). The curve is linear. However for small values of $\varepsilon$, it is linear and gives the value of $\alpha_{\mathrm{p}}$ as $0.7 * 10^{2} *\left(10^{-24} \mathrm{~cm}^{3}\right)$ for polarizability which is defined as

Table:

\begin{tabular}{|c|c|c|c|c|c|c|}
\hline \multirow{2}{*}{$\beta^{-1 / 2}$} & \multirow{2}{*}{$(\mathbf{n m})$} & \multicolumn{5}{|c|}{$\left\langle\mathrm{E}>\right.$ in $\mathrm{Ryd}^{*}$} \\
\hline & & $\varepsilon=0$ & $\varepsilon=10$ & $\varepsilon=20$ & $\varepsilon=40$ & $\varepsilon=60$ \\
\hline 2 & & 13.18 & 13.48 & 13.47 & 13.46 & 13.44 \\
\hline 4 & & 12.10 & 12.42 & 12.39 & 12.35 & 12.27 \\
\hline 6 & & 7.93 & 8.16 & 8.12 & 8.10 & 8.02 \\
\hline 8 & & 4.99 & 5.42 & 5.39 & 5.37 & 5.26 \\
\hline 10 & & 3.18 & 3.57 & 3.49 & 3.46 & 3.38 \\
\hline
\end{tabular}

$* 1 \mathrm{Ryd}=5.3 \mathrm{meV}$ for Ga As

$\alpha_{\mathrm{p}}=\left.\left[\partial<\mathrm{E}>/ \partial \varepsilon^{2}\right]\right|_{\varepsilon=>0}=0.7 * 10^{2} *\left(10^{-24} \mathrm{~cm}^{3}\right)$

\section{Conclusion.}

We have presented a calculation for the binding energy of the ground state for a shallow hydrogenic donor in spherical GaAs QDs with parabolic confinement, following variational procedure within the effective mass approximations. The computed result shows that the binding energy increases as the dot size decreases. In spherical quantum dots, with an isotropic parabolic potential, the impurity binding energy is found to decrease as the impurity moves away from the center, the effect being more pronounced for dots of smaller sizes.

\section{Reference Books:}

[1] Bastard G 1981 Phys.Rev.B 244714

[2] Chayanika Bose, 1998 J,Appl. Phys. 833089

[3] Sakaki H 1981 J.Vac.Sci. Technol. 19148

[4] Vojak B.A., Laidig W.D, Holonyak N.Camras M D, Coleman J J and Dapkus

[5] Schiff. L. I. (1968) Quantum Mechanics, III rd Edition, Phys.265

[6] Lorke, J.P.Kotthaus, and K.Ploog, Phys.Rev. Lett. 64 , 2559(1990)

[7] M.J.Kelly, Low dimensional semiconductors: Materials, Physics technology,Devices(Oxford: Calrendon 1995) and references therein

[8] F.J.Ribeiro and A.Latge, Phys.Rev.50 (1994) 4913.

[9] J.Silva-Valencia and N.Porras- Montenegro, J.Appl.Phys. 81 (1997) 901.

[10] D.J.Wolford and J.A.Bradly, Solid State Commun. 53 (1985) 1069

[11] H.O.Oyoko, C.A. Duque, and N. Porras-Montenegro, J.Appl.Phys. 90 (2001) 819. 